Further characterization of these proteins indicates that the disorganized patterning of tumour blood vessels might reflect the different actions of VEGF. Soluble VEGF (VEGF ${ }_{113}$ ) that is present in discrete patches where MMPs are active induces proliferation of endothelial cells, resulting in dilated blood vessels, whereas ECM-bound VEGF induces the active sprouting and branching of new blood vessels from existing ones.

These findings illustrate that the effect of VEGF during angiogenesis is complex and the availability and action of MMPs in tumours might go some way to explain the heterogeneous pattern of tumour blood vessels. Importantly, however, as the VEGF tumours $_{113}$ did not grow well, it is unlikely that circulating levels of VEGF will correlate with tumour progression. Therefore, VEGF levels might not be a suitable prognostic factor.

Nicola McCarthy

(2) References and links

ORIGINAL RESEARCH PAPER Lee, $S$. et al. Processing of VEGF-A by matrix metalloproteinases regulates bioavailability and vascular patterning in tumours. J. Cell Biol. 23 May 2005 (doi:10.1083/jbc.200409115)

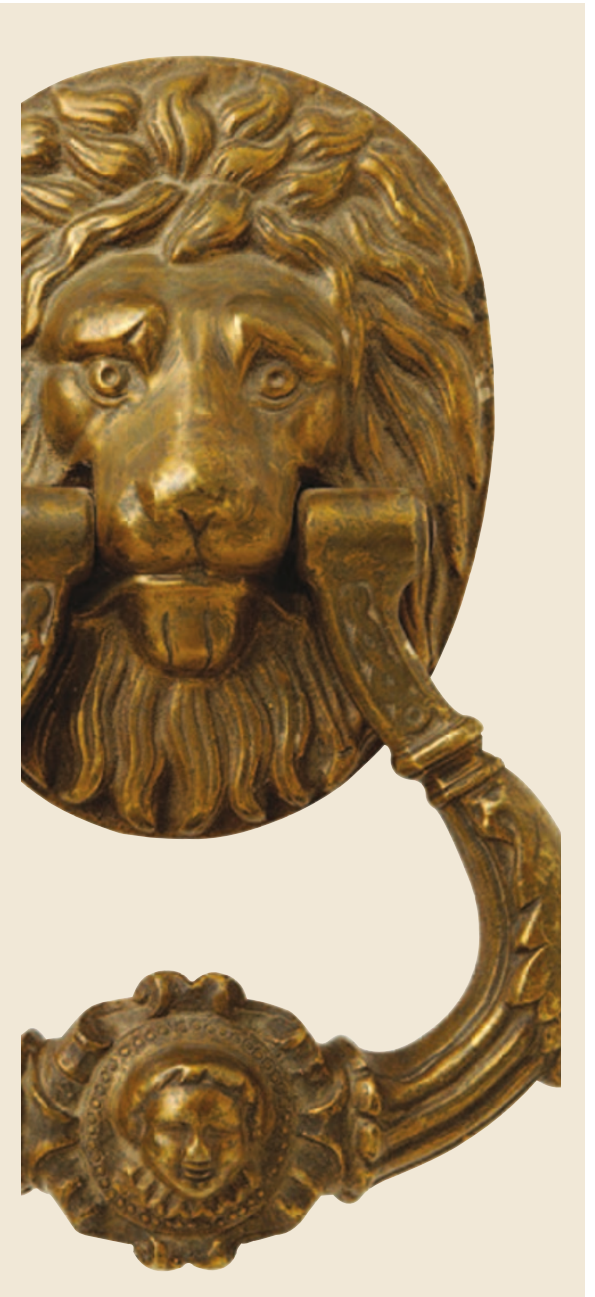

IMMUNOLOGY

\title{
B cells lead the way in tumour progression
}

Many studies have reported a link between chronic inflammatory diseases and cancer development. However, it has not been clear how the recruitment of inflammatory innate immune cells is initiated or sustained at sites of tumour development. Lisa Coussens and colleagues now report an important role for $B$ cells in promoting innate-immunecell inflammation in a mouse model of epithelial carcinogenesis, which is in contrast to the view that adaptive immune cells are involved in 'surveillance' against developing neoplasms.

K14-HPV16 mice express oncogenes from human papillomavirus type 16 under the control of the human keratin 14 promoter/enhancer, leading to multistage epithelial carcinogenesis. The pre-malignant stage is characterized by infiltration of innate immune cells such as granulocytes and mast cells into the skin. However, when K14-HPV16 mice were crossed with recombinationactivating-gene-1-deficient mice (K14HPV16/Rag1 ${ }^{-/-}$mice), which lack $\mathrm{T}$ and $B$ cells, this infiltration was significantly reduced. Decreased infiltration of the skin was associated with decreased activity of matrix metalloproteinase 9 (MMP9) compared with K14-HPV16 mice. MMP9 is secreted by leukocytes and has a role in cancer development through its effects on tissue remodelling and release of the angiogenic growth factor vascular endothelial growth factor (VEGF) from the extracellular matrix. Correspondingly, K14-HPV16/Rag1 ${ }^{-/-}$mice had reduced levels of VEGF in skin lysates compared with K14-HPV16 mice, as well as decreased markers of angiogenesis.

The lack of typical pre-malignant inflammatory characteristics in K14HPV16/Rag1 ${ }^{-1-}$ mice was associated with decreased progression to epithelial carcinoma. Only $6.4 \%$ of K14-HPV16/ Rag1 $^{-/-}$mice developed full-blown carcinomas compared with $47 \%$ of K14HPV16 mice. Therefore, the lack of $\mathrm{T}$ and $B$ cells of the adaptive immune system inhibits pre-malignant inflammation and tumour progression in this model.

To examine the specific role of $B$ cells in this process, the authors looked at antibody deposition. B cells do not infiltrate pre-malignant skin, but they might exert their effects through the systemic production of antibodies specific for antigens in the skin. Deposits of immunoglobulin G (IgG) and IgM could be detected in the skin of K14HPV 16 mice by 1 month of age, and these increased up to 6 months of age in association with the development of chronic inflammation. The adoptive transfer of B cells from K14-HPV16 mice, which would contain primed and/or memory B cells of the desired specificity,

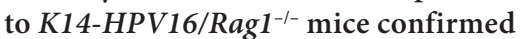
the important role of $B$ cells by restoring leukocyte infiltration of the skin and other downstream characteristics such as angiogenesis. The transfer of serum from K14-HPV16 mice had a similar effect, which is in line with the postulated systemic actions of B cells through the production of antibodies and/or soluble mediators such as cytokines.

The authors conclude that $\mathrm{B}$ cells are a crucial part of tumour progression in this model through the promotion of chronic inflammation in the pre-malignant state. Therefore, therapies that aim to stimulate B-cell responses, such as vaccination, should be used with caution in cancer-prone patients or patients with pre-malignant disease.

Kirsty Minton

Associate Editor, Nature Reviews Immunology

\section{Deferences and links}

ORIGINAL RESEARCH PAPER de Visser, K. E., Korets, L. V. $\&$ Coussens, L. M. De novo carcinogenesis promoted by chronic inflammation is B lymphocyte dependent. Cancer Cell $\mathbf{7}$, 411-423 (2005)

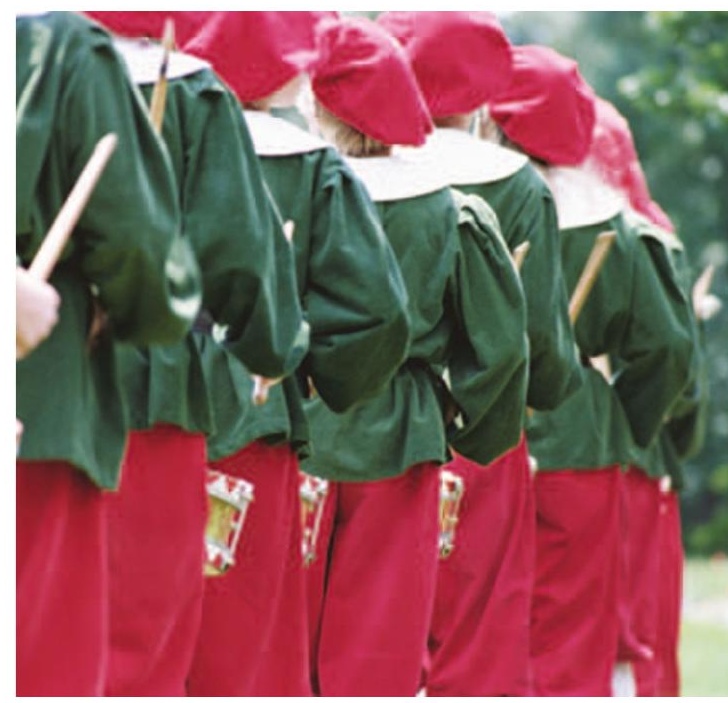

